\title{
Control of LPV Systems using a Quasi-Piecewise Affine Parameter-Dependent Lyapunov Function
}

\author{
Sungyung Lim ${ }^{1}$ and Jonathan P. How ${ }^{2}$ \\ Dept. of Aeronautics and Astronautics \\ Stanford University, Stanford, CA 94305
}

\begin{abstract}
This paper presents a new finite-dimensional linear matrix inequality (LMI) formulation for the induced $L_{2}$-norm synthesis of linear parameter-varying (LPV) systems. The approach is based on a nonsmooth dissipative systems theory using a continuous, quasi-piecewise affine parameter-dependent Lyapunov function. The new method is less conservative than previously published techniques based on either affine parameter-dependent Lyapunov functions or robust control techniques. Conservatism is reduced with this new approach because the synthesis uses a very general class of parameterdependent Lyapunov functions. In contrast to the gridding approach typically used to develop a computationally feasible algorithm, this proposed approach guarantees the synthesis result. We show that the numerical results using our approach, while computationally intensive, can be used to develop many new insights into the potential conservatism of various classes of Lyapunov functions for LPV systems.
\end{abstract}

\section{Introduction}

Linear parameter-varying (LPV) systems have recently received much attention because they provide a systematic means of computing gain-scheduled controllers [14,15]. Various synthesis tools have been proposed for LPV systems such as the LFT- $\mu$ framework $[2,9,12]$ and the dissipative systems theory with smooth parameter-dependent Lyapunov functions $[6,16]$, the selection of which determines the conservatism of the synthesis result. The selection of an appropriate Lyapunov function is one of the most important issues in the dissipative systems theory. The class of Lyapunov function used in the LPV framework is of the form, $V=x_{\mathrm{cl}}^{T} P_{\mathrm{cl}}(\theta) x_{\mathrm{cl}}$, such that

$$
P_{\mathrm{cl}}(\theta)=\left[\begin{array}{cc}
X(\theta) & Z(\theta) \\
Z^{T}(\theta) & E(\theta)
\end{array}\right] \quad\left(P_{\mathrm{cl}}^{-1}(\theta)=\left[\begin{array}{cc}
Y(\theta) & * \\
* & *
\end{array}\right]\right) .
$$

The classes of quadratic [4] and affine parameter-dependent Lyapunov functions (ALFs) have been widely used in the literature. In the class of ALFs, $X(\theta)$ is typically affine in $\theta$, and $Z(\theta)$ and $E(\theta)$ are constant $[3,6]$. This type is a special form of the Luré-Postnikov Lyapunov function. The synthesis using this ALF is formulated as a bilinear matrix inequality (BMI) problem, which is computationally intensive and often yields a parameter-independent controller. In another class of a Lyapunov function, quasi-affine parameter-dependent Lyapunov function (QAL), $X(\theta)$ and $Y(\theta)$ are affine in $\theta$ [1]. The synthesis using this QAL leads to linear matrix inequality (LMI) formulations and yields a parameter-dependent con-

\footnotetext{
${ }^{1} \mathrm{Ph} . \mathrm{D}$. Candidate, limsy@sun-valley.stanford.edu

${ }^{2}$ Assistant Professor, howjo@sun-valley.stanford.edu
}

troller. While QAL is more general than ALF, a more general class of Lyapunov functions than QAL is still required to reduce conservatism even further. Unfortunately, the synthesis using this type of Lyapunov functions often leads to infinite-dimensional LMIs. As a result, the finite basis methods and gridding techniques [16] are typically used. However, these techniques do not guarantee the synthesis result, and the selection of the best basis function requires further study.

This paper extends our previous result [11] to address the synthesis of controllers for LPV systems and generalizes it to a new class of parameter-dependent Lyapunov functions. We then develop a new synthesis technique that reduces the conservatism but still leads to finite-dimensional LMI formulations. Our approach is to a priori partition the entire parameter space into several smaller subspaces. We then define an affine parameter-dependent LPV (ALPV) system and QAL over each parameter subspace. Constraints are included so that these QALs define a continuous, Lipschitz Lyapunov function over the entire parameter space, which is called a quasi-piecewise affine parameter-dependent Lyapunov function (QPAL). This QPAL is then used to design a $\gamma$-suboptimal LPV controller for this piecewise-affine parameter-dependent LPV (PALPV) system. Numerical results are presented to show that, even with a smaller number of partitions, this new technique yields less conservative results than previously published research $[1,3]$. Furthermore, in contrast to the gridding approach of Ref. [16], our technique guarantees the synthesis result. The computational burden of our approach currently limits the complexity of the examples that can be tested, but the initial results indicate that this technique could be used to investigate numerically good basis functions for Ref. [16].

\section{Notations and Problem Definitions}

Some notations and definitions for this paper are established. $\mathcal{I}=\left[t_{0}, \infty\right)$ and the set of disturbance is $\mathcal{W} \subset \mathcal{L}_{2}\left(\mathcal{I}, \mathcal{R}^{n_{w}}\right)$, which is square integrable $\mathcal{R}^{n_{w}}$ vector sets over $\mathcal{I}$. The compact set of state, $\mathcal{D} \subset \mathcal{R}^{2 n}$, includes the origin $x(t)=0$. The parameter set

$$
\mathcal{F}^{s}=\left\{\theta \in C^{1}\left(\mathcal{I}, \mathcal{R}^{s}\right): \theta(t) \in \mathcal{P}, \dot{\theta}(t) \in \Omega, \forall t \in \mathcal{I}\right\}
$$

where $\mathcal{P}=\left[\underline{\theta}_{1}, \bar{\theta}_{1}\right] \times \cdots \times\left[\underline{\theta}_{s}, \bar{\theta}_{s}\right]$ and $\Omega=\left[-\nu_{1}, \nu_{1}\right] \times \cdots \times$ $\left[-\nu_{s}, \nu_{s}\right] . \quad \operatorname{Co}[x, y]_{\lambda} \triangleq\{z \mid z=(1 / 2-\lambda) x+(1 / 2+\lambda) y\}$, for $-1 / 2 \leq \lambda \leq 1 / 2 .[A(\theta)]_{i j} \triangleq\left[A_{i j}\left(\theta_{i j}\right)\right] .\|u\|_{2}^{2}=\int_{0}^{\infty} u^{T} u d t$. The dini-derivatives of $V(x(t), \theta(t))$ are defined as follows:

$$
\begin{gathered}
D^{+} V(x, \theta)=\lim _{h \rightarrow 0^{+}} \sup \frac{1}{h}[V(x(t+h), \theta(t+h))-V(x(t), \theta(t))], \\
D^{+} V(x, \theta)(u, 0)=\lim _{h \rightarrow 0^{+}} \sup \frac{1}{h}[V(x+h u, \theta)-V(x, \theta)],
\end{gathered}
$$




$$
D_{+} V(x, \theta)(u, 0)=\lim _{h \rightarrow 0^{+}} \inf \frac{1}{h}[V(x+h u, \theta)-V(x, \theta)] .
$$

For simplicity, we consider the case of $s=2$ such that the parameter space $\mathcal{P} \subset \mathcal{R}^{2}$. The $\mathcal{P}$ is partitioned into $m_{1} \times$ $m_{2}$ identical, closed rectangles, $\mathcal{P}_{i j}$ 's, with width $\Delta \theta_{1} \times \Delta \theta_{2}$. In this case, $\Delta \theta_{k}=\left(\bar{\theta}_{k}-\underline{\theta}_{k}\right) / m_{k}$ for $k=1,2$. A PALPV system is defined as a system that switches between $m_{1} \times$ $m_{2}$ ALPV systems. Each ALPV system is described by a set of nominal dynamics at the center of the corresponding parameter subspace and affine parameter-dependent terms. For each subspace $\mathcal{P}_{i j}$, we introduce a local coordinate $\hat{\theta}_{i j}=$ $\left[\hat{\theta}_{i j 1} \hat{\theta}_{i j 2}\right]^{T}$ measured from the center of $\mathcal{P}_{i j}$. As a result, this PALPV can be defined such that for $\theta(t) \in \mathcal{F}^{2}$,

$$
\begin{gathered}
{\left[\begin{array}{c}
\dot{x} \\
z_{1} \\
z_{2} \\
y
\end{array}\right]=\sum_{i=1}^{m_{1}} \sum_{j=1}^{m_{2}} \alpha_{i j}(\theta) \mathcal{G}_{i j}\left[\begin{array}{c}
x \\
w_{1} \\
w_{2} \\
u
\end{array}\right],} \\
\mathcal{G}_{i j}=\left[\begin{array}{c|ccc}
A(\hat{\theta}) & B_{w 1}(\hat{\theta}) & B_{w 2} & B_{u}(\hat{\theta}) \\
\hline C_{z 1}(\hat{\theta}) & 0 & 0 & 0 \\
C_{z 2} & 0 & 0 & I_{n_{u}} \\
C_{y}(\hat{\theta}) & 0 & I_{n_{y}} & 0
\end{array}\right]_{i j}
\end{gathered}
$$

for $\hat{\theta}_{i j}(t) \in \hat{\mathcal{P}} \triangleq\left[-\frac{\Delta \theta_{1}}{2}, \frac{\Delta \theta_{1}}{2}\right] \times\left[-\frac{\Delta \theta_{2}}{2}, \frac{\Delta \theta_{2}}{2}\right]$ and $\dot{\hat{\theta}}_{i j}(t)=\dot{\theta} \in$ $\Omega$. Here, all $\hat{\theta}_{i j}$-dependent system matrices are affine dependent, e.g., $A_{i j}\left(\hat{\theta}_{i j}\right)=A_{i j 0}+\sum_{k=1}^{2} \hat{\theta}_{i j k} A_{i j k}$. It is assumed that $\theta(t)$ and $\dot{\theta}(t)$ are measurable in real time. $\alpha_{i j}(\theta)$ is the switching function such that each $\mathcal{G}_{i j}$ is well defined over the corresponding $\mathcal{P}_{i j}[11]$.

Remark 1: The PALPV system (Eq. 1) is locally affine parameter-dependent. The typical ALPV system description is a special case of this PALPV description. Our representation can include dynamics that are discontinuous in $\theta(t)$ on the boundary of $\mathcal{P}_{i j}$, which could provide better approximation models for general LPV systems.

Our problem is to find a strictly proper full-order LPV controller of the form

$$
\left[\begin{array}{c}
\dot{x}_{c} \\
u
\end{array}\right]=\sum_{i=1}^{m_{1}} \sum_{j=1}^{m_{2}} \alpha_{i j}(\theta)\left[\begin{array}{cc}
A_{c}(\hat{\theta}, \dot{\hat{\theta}}) & B_{c}(\hat{\theta}, \dot{\hat{\theta}}) \\
C_{c}(\hat{\theta}, \hat{\hat{\theta}}) & 0
\end{array}\right]_{i j}\left[\begin{array}{c}
x_{c} \\
y
\end{array}\right] .
$$

where $\left[A_{c}, B_{c}, C_{c}\right]_{i j}$ are continuous over $\mathcal{P}_{i j}$. With the notations of $x_{c 1}^{T}=\left[\begin{array}{ll}x^{T} & x_{c}^{T}\end{array}\right], w^{T}=\left[\begin{array}{ll}w_{1}^{T} & w_{2}^{T}\end{array}\right]$ and $z^{T}=\left[\begin{array}{ll}z_{1}^{T} & z_{2}^{T}\end{array}\right]$, the closed-loop system can be then written as

$$
\left[\begin{array}{c}
\dot{x}_{\mathrm{cl}} \\
z
\end{array}\right]=\sum_{i=1}^{m_{1}} \sum_{j=1}^{m_{2}} \alpha_{i j}(\theta)\left[\begin{array}{cc}
A_{\mathrm{cl}}(\hat{\theta}, \dot{\hat{\theta}}) & B_{\mathrm{cl}}(\hat{\theta}, \dot{\hat{\theta}}) \\
C_{\mathrm{cl}}(\hat{\theta}, \hat{\hat{\theta}}) & 0
\end{array}\right]_{i j}\left[\begin{array}{c}
x_{\mathrm{cl}} \\
w
\end{array}\right] .
$$

\section{Induced $L_{2}$-Norm Synthesis}

The problem we consider here is to design $\gamma$-suboptimal controller such that the induced $L_{2}$-norm of the closed-loop system (Eq. 3) is less than $\gamma$, i.e., $\|z(t)\|_{2}<\gamma\|w(t)\|_{2}$, where $\gamma=\sup _{\theta \in \mathcal{F}^{2}} \sup _{w \in \mathcal{W}} \frac{\|z(t)\|_{2}}{\|w(t)\|_{2}}$. The synthesis typically consists of three steps: [Step 1] Formulate the analysis problem of the closed-loop system; [Step 2a] Eliminate unknown controller dynamics from the analysis formulation; [Step 2b] If necessary, "convexify" the formulation from Step 2a to transform

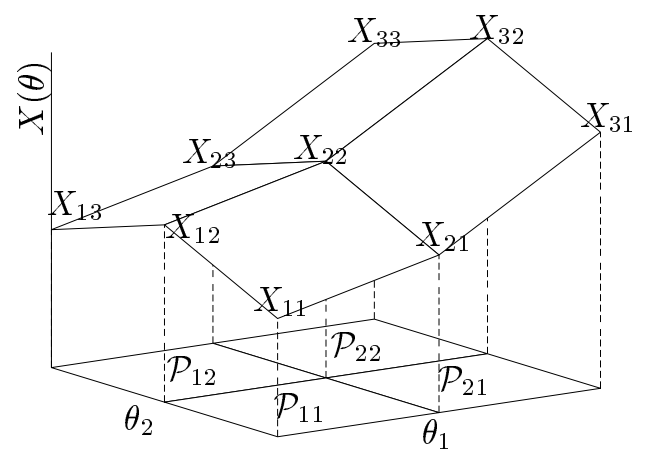

Fig. 1: Example of a continuous, piecewise affine parameter-dependent $X(\theta) \in \mathcal{R}$

into finite-dimensional LMIs and then solve them; and [Step 3] Construct a controller dynamics analytically or numerically.

Step 1: A nonsmooth dissipative systems theory for analysis problems has been proposed in our previous work [11]. We consider a quasi-piecewise affine parameter-dependent Lyapunov function (QPAL), $V\left(x_{\mathrm{cl}}, \theta\right)=x_{\mathrm{cl}}^{T} P_{\mathrm{cl}}(\theta) x_{\mathrm{cl}}$, such that

$$
P_{\mathrm{cl}}(\theta)=\sum_{i=1}^{m_{1}} \sum_{j=1}^{m_{2}} \alpha_{i j}(\theta) P_{i j}\left(\hat{\theta}_{i j}\right)
$$

$P_{i j}\left(\hat{\theta}_{i j}\right)=\left[\begin{array}{cr}\mathcal{X}(\hat{\theta}) & -\mathcal{X}(\hat{\theta})+\gamma^{-2} \mathcal{Y}^{-1}(\hat{\theta}) \\ -\mathcal{X}(\hat{\theta})+\gamma^{-2} \mathcal{Y}^{-1}(\hat{\theta}) & \mathcal{X}(\hat{\theta})-\gamma^{-2} \mathcal{Y}^{-1}(\hat{\theta})\end{array}\right]_{i j}$.

Here, $\mathcal{X}_{i j}\left(\hat{\theta}_{i j}\right)=\operatorname{Co}\left[X_{i j}, X_{(i+1) j}, X_{(i+1)(j+1)}, X_{i(j+1)}\right]_{\hat{\theta}_{i j}}$, whose vertices are positive matrices defined counterclockwise from the corner of the rectangle $\mathcal{P}_{i j}$ (see Figure 1 for $\left.X(\theta)=\sum_{i=1}^{m_{1}} \sum_{j=1}^{m_{2}} \alpha_{i j} \mathcal{X}_{i j}\left(\hat{\theta}_{i j}\right)\right)$. To ensure that $\mathcal{X}_{i j}\left(\hat{\theta}_{i j}\right)$ over each $\mathcal{P}_{i j}$ is affine parameter-dependent, these matrices must satisfy

$$
X_{i j}+X_{(i+1)(j+1)}=X_{(i+1) j}+X_{i(j+1)}
$$

which is one of the parallelogram rules. Substituting Eq. 5,

$$
\begin{aligned}
\mathcal{X}_{i j}\left(\hat{\theta}_{i j}\right)= & X_{i j 0}+\sum_{k=1}^{2} \hat{\theta}_{i j k} X_{i j k} \\
= & \frac{1}{2}\left(X_{(i+1) 1}+X_{i 1}+X_{1(j+1)}+X_{1 j}-2 X_{11}\right) \\
& +\hat{\theta}_{i j 1} \frac{X_{(i+1) 1}-X_{i 1}}{\Delta \theta_{1}}+\hat{\theta}_{i j 2} \frac{X_{1(j+1)}-X_{1 j}}{\Delta \theta_{2}} .
\end{aligned}
$$

$\mathcal{Y}_{i j}\left(\hat{\theta}_{i j}\right)$ is also similarly defined in terms of $Y_{i j}$ 's.

Remark 2: Note that while possibly conservative, this specific structure of $P_{\mathrm{cl}}(\theta)$ forces $(1,1)$ element of $P_{\mathrm{cl}}^{-1}(\theta)$ to be $\gamma^{2} Y(\theta)$, so that the $(1,1)$ elements of $P_{\mathrm{cl}}(\theta)$ and $P_{\mathrm{cl}}^{-1}(\theta)$ are piecewise affine parameter-dependent. Note, also, that the similar form of $P_{\mathrm{cl}}(\theta)$ with $m_{1}=m_{2}=1$ can be found in [16]

Remark 3: Over each $\mathcal{P}_{i j}, P_{i j}\left(\hat{\theta}_{i j}\right)$ is a differentiable matrix function because $\mathcal{Y}_{i j}^{-1}\left(\hat{\theta}_{i j}\right)$ is a rational matrix function which is differentiable. By sharing some vertices between $\mathcal{X}_{i j}\left(\hat{\theta}_{i j}\right)$ 's and $\mathcal{Y}_{i j}\left(\hat{\theta}_{i j}\right)$ 's, $P_{\mathrm{cl}}(\theta)$ becomes a globally continuous matrix function over $\mathcal{P}$. As a result, $P_{\mathrm{cl}}(\theta)$ does have the dini-derivative over $\mathcal{P}$, thereby being Lipschitz in $\theta$.

Using the QPAL, we can derive the induced $L_{2}$-norm analysis formulation for the closed-loop system from the result of [11]. 
Proposition 1: Suppose there exist differentiable functions $P_{i j}\left(\hat{\theta}_{i j}\right)>0$ on $\mathcal{P}_{i j}$ for $\forall i, j$ such that

$$
\left[\begin{array}{ccc}
A_{\mathrm{cl}}^{T} P+P A_{\mathrm{cl}}+\frac{\partial P}{\partial \hat{\theta}} \dot{\hat{\theta}} & P B_{\mathrm{cl}} & \gamma^{-1} C_{\mathrm{cl}}^{T} \\
B_{\mathrm{cl}}^{T} P & -I & 0 \\
\gamma^{-1} C_{\mathrm{cl}} & 0 & -I
\end{array}\right]_{i j}<0
$$

for all $\hat{\theta}_{i j} \in \hat{\mathcal{P}}$ and $\dot{\hat{\theta}}_{i j} \in \Omega$. Then for any $\theta(t) \in \mathcal{F}^{2}$, the closed-loop system (Eq. 3) is uniformly asymptotically stable and its $L_{2}$-norm is bounded by $\gamma$, i.e., $\|z\|_{2}<\gamma\|w\|_{2}$.

Proof: Consider $V\left(x_{\mathrm{cl}}, \theta\right)=x_{\mathrm{cl}}^{T} P_{\mathrm{cl}}(\theta) x_{\mathrm{cl}}$ such that $P_{\mathrm{cl}}(\theta)$ is defined as Eq. 4. As in [11], the sufficient conditions to guaranteed $\gamma$ performance of Eq. 3 are (I) $P_{\mathrm{cl}}(\theta)>0$ and

$$
\text { (II) } D^{+} V\left(x_{\mathrm{cl}}, \theta\right)+z^{T} z-\gamma^{2} w^{T} w<0 \text {. }
$$

Condition (I) obviously means $P_{i j}\left(\hat{\theta}_{i j}\right)>0$, for $\forall i, j$. Consider condition (II). According to Lemma 2 in the appendix,

$$
D^{+} V\left(x_{\mathrm{cl}}, \theta\right) \leq D^{+} V\left(x_{\mathrm{cl}}, \theta\right)(U, 0)+D^{+} V\left(x_{\mathrm{cl}}, \theta\right)(0, \dot{\theta})
$$

for $\forall x_{\mathrm{cl}} \in \mathcal{D}, \theta \in \mathcal{P}$ and $\dot{\theta} \in \Omega$. Here,

$$
U(t)=\lim _{h \rightarrow 0} \sup \left[\mathcal{S}_{h}(t)=\{y \mid y=\dot{x}(t+\tau),-h \leq \tau \leq h\}\right] .
$$

To calculate this "partial" dini-derivative, this inequality over the domain $\mathcal{D} \times \mathcal{P} \times \Omega$ is divided into $m_{1} \times m_{2}$ inequalities over sub-domains $\mathcal{D} \times \mathcal{P}_{i j} \times \Omega$ for all $i, j$, as in [11]. Note that this is accomplished by the assumption that, by a smooth extension, the system dynamics (Eq. 3) and the Lyapunov function (Eq. 4) are defined over the smallest open set that includes $\mathcal{P}_{i j}$. This assumption makes $V\left(x_{\mathrm{cl}}, \theta\right)$ differentiable on each $\mathcal{P}_{i j}$ but may introduce some conservatism. Therefore, condition (II) is overbounded by

$$
\frac{\partial\left[x_{\mathrm{cl}}^{T} P_{i j} x_{\mathrm{cl}}\right]}{\partial x_{\mathrm{cl}}} \dot{x}_{\mathrm{cl}}+\frac{\partial\left[x_{\mathrm{cl}}^{T} P_{i j} x_{\mathrm{cl}}\right]}{\partial \hat{\theta}_{i j}} \dot{\hat{\theta}}_{i j}+z^{T} z-\gamma^{2} w^{T} w<0
$$

for $\forall\left(x_{\mathrm{cl}}, \hat{\theta}_{i j}, \dot{\hat{\theta}}_{i j}, w\right) \in \mathcal{D} \times \hat{\mathcal{P}} \times \Omega \times \mathcal{W}$ and $\forall i, j$. Substituting Eqs. $3-4$, condition (II) is then

$$
\begin{aligned}
& 2 x_{\mathrm{cl}}^{T} P_{i j}\left[A_{\mathrm{cl} i j} x_{\mathrm{cl}}+B_{\mathrm{cl} i j} w\right]+\sum_{k=1}^{2} x_{\mathrm{cl}}^{T} \dot{\hat{\theta}}_{i j k} \frac{\partial P_{i j}}{\partial \dot{\hat{\theta}}_{i j k}} x_{\mathrm{cl}} \\
& +\left[C_{\mathrm{cl} i j} x_{\mathrm{cl}}\right]^{T}\left[C_{\mathrm{cl} i j} x_{\mathrm{cl}}\right]-\gamma^{2} w^{T} w<0
\end{aligned}
$$

for $\forall\left(x_{\mathrm{cl}}, \hat{\theta}_{i j}, \dot{\hat{\theta}}_{i j}, w\right) \in \mathcal{D} \times \hat{\mathcal{P}} \times \Omega \times \mathcal{W}$ and $\forall i, j$. Divide both sides by $\gamma^{2}$ and reformulate the above inequality as the standard quadratic matrix form.

Step 2 \& Step 3: Over each $\mathcal{P}_{i j}$, Proposition 1 is exactly equal to the typical induced $L_{2}$-norm analysis using a continuously differentiable Lyapunov function. According to the result of [16], LMI formulations without any controller dynamics and an explicit formula for a $\gamma$-suboptimal LPV controller are easily found (For details, refer to [16]).

Lemma 1: The set of $\gamma$-suboptimal controllers is nonempty if and only if there exist symmetric positive matrices $\mathcal{X}_{i j}\left(\hat{\theta}_{i j}\right)$, $\mathcal{Y}_{i j}\left(\hat{\theta}_{i j}\right)$ for $\forall i, j$ such that for $\forall \hat{\theta}_{i j} \in \hat{\mathcal{P}}$ and $\dot{\hat{\theta}}_{i j} \in \Omega$,

$$
\left[\begin{array}{ll}
\mathcal{X}(\hat{\theta}) & \gamma^{-1} I \\
\gamma^{-1} I & \mathcal{Y}(\hat{\theta})
\end{array}\right]_{i j}>0
$$

$$
\left[\begin{array}{ccc}
\mathcal{Y}(\hat{\theta}) \hat{A}^{T}(\hat{\theta})+\hat{A}(\hat{\theta}) \mathcal{Y}(\hat{\theta}) & \mathcal{Y}(\hat{\theta}) C_{z 1}^{T}(\hat{\theta}) & \gamma^{-1} B_{w}(\hat{\theta}) \\
+\frac{\partial \mathcal{Y}(\hat{\theta})}{\partial \hat{\theta}} \cdot \hat{\theta}-B_{u}(\hat{\theta}) B_{u}^{T}(\hat{\theta}) & & \\
C_{z 1}(\hat{\theta}) \mathcal{Y}(\hat{\theta}) & -I & 0 \\
\gamma^{-1} B_{w}^{T}(\hat{\theta}) & 0 & -I
\end{array}\right]_{i j}<0
$$$$
\left[\begin{array}{ccc}
\tilde{A}^{T}(\hat{\theta}) \mathcal{X}(\hat{\theta})+\mathcal{X}(\hat{\theta}) \tilde{A}(\hat{\theta}) & \mathcal{X}(\hat{\theta}) B_{w 1}(\hat{\theta}) & \gamma^{-1} C_{z}^{T}(\hat{\theta}) \\
+\frac{\partial \mathcal{X}(\hat{\theta})}{\partial \hat{\theta}} \dot{\hat{\theta}}-C_{y}^{T}(\hat{\theta}) C_{y}(\hat{\theta}) & & \\
B_{w 1}^{T}(\hat{\theta}) \mathcal{X}(\hat{\theta}) & -I & 0 \\
\gamma^{-1} C_{z}(\hat{\theta}) & 0 & -I
\end{array}\right]_{i j}<0
$$

Here, $\hat{A}_{i j}\left(\hat{\theta}_{i j}\right)=A_{i j}\left(\hat{\theta}_{i j}\right)-B_{u i j}\left(\hat{\theta}_{i j}\right) C_{z 2}, \quad B_{w i j}\left(\hat{\theta}_{i j}\right)=$ $\left[\begin{array}{ll}B_{w 1 i j}\left(\hat{\theta}_{i j}\right) & B_{w 2}\end{array}\right], \tilde{A}_{i j}\left(\hat{\theta}_{i j}\right)=A_{i j}\left(\hat{\theta}_{i j}\right)-B_{w 2} C_{y i j}\left(\hat{\theta}_{i j}\right)$ and $C_{z i j}^{T}\left(\hat{\theta}_{i j}\right)=\left[C_{z 1 i j}^{T}\left(\hat{\theta}_{i j}\right) C_{z 2}^{T}\right]$. Over each $\mathcal{P}_{i j}$, an explicit formula for a central strictly proper controller $\left[A_{c}, B_{c}, C_{c}\right]_{i j}$ is given at Theorem 5.3.1 of [16].

"Convexifying": Eqs. 11 and 12 are not finite-dimensional LMIs because they includes the cross-product terms such as $\mathcal{X}_{i j}\left(\hat{\theta}_{i j}\right) \tilde{A}_{i j}\left(\hat{\theta}_{i j}\right)$. As in the previous work [6,11], we apply the quadratic relation and $\mathcal{S}$-procedure to Eqs. 11 and 12 to derive finite-dimensional LMIs. Note, however, that this "convexifying" technique often introduces conservatism and increases the problem size (refer to [11]). To simplify the notation, we introduce the following matrices: $\hat{A}_{i j}=\hat{A}_{i j 0}+$ $\sum_{k=1}^{2} \hat{\theta}_{i j k} \hat{A}_{i j k}, \tilde{A}_{i j}=\tilde{A}_{i j 0}+\sum_{k=1}^{2} \hat{\theta}_{i j k} \tilde{A}_{i j k}, B_{w i j}=B_{w i j 0}+$ $\sum_{k=1}^{2} \hat{\theta}_{i j k} B_{w i j k}, C_{z i j}=C_{z i j 0}+\sum_{k=1}^{2} \hat{\theta}_{i j k} C_{z i j k}, \mathcal{I}^{T}=\left[\begin{array}{ll}I & I\end{array}\right]$,

$$
\begin{aligned}
& \hat{\mathcal{A}}_{i j}^{T}=\left[\begin{array}{ll}
\hat{A}_{i j 1}^{T} & \hat{A}_{i j 2}^{T}
\end{array}\right] \quad \tilde{\mathcal{A}}_{i j}=\left[\begin{array}{ll}
\tilde{A}_{i j 1} & \tilde{A}_{i j 2}
\end{array}\right] \\
& \hat{B}_{w i j}^{T}=\left[\begin{array}{ll}
B_{w i j 1}^{T} & B_{w i j 2}^{T}
\end{array}\right] \quad \tilde{B}_{w 1 i j}=\left[\begin{array}{ll}
B_{w 1 i j 1} & B_{w 1 i j 2}
\end{array}\right] \\
& \hat{B}_{u i j}^{T}=\left[\begin{array}{ll}
B_{u i j 1}^{T} & B_{u i j 2}^{T}
\end{array}\right] \quad \tilde{C}_{z i j}=\left[\begin{array}{ll}
C_{z i j 1} & C_{z i j 2}
\end{array}\right] \\
& \hat{C}_{z 1 i j}^{T}=\left[\begin{array}{ll}
C_{z 1 i j 1}^{T} & C_{z 1 i j 2}^{T}
\end{array}\right] \quad \tilde{C}_{y i j}=\left[\begin{array}{ll}
C_{y i j 1} & C_{y i j 2}
\end{array}\right] \\
& \hat{Y}_{i j}=\left[\begin{array}{ll}
Y_{i j 1} & Y_{i j 2}
\end{array}\right] \quad \tilde{X}_{i j}=\left[\begin{array}{ll}
X_{i j 1} & X_{i j 2}
\end{array}\right] \\
& \hat{T}_{i j}=\left[\begin{array}{ll}
T_{i j 1} & T_{i j 2}
\end{array}\right] \quad \tilde{T}_{i j}=\left[\begin{array}{ll}
T_{i j 1} & T_{i j 2}
\end{array}\right] \\
& \hat{V}_{i j}=\left[\begin{array}{ll}
V_{i j 1} & V_{i j 2}
\end{array}\right] \quad \tilde{V}_{i j}=\left[\begin{array}{ll}
V_{i j 1} & V_{i j 2}
\end{array}\right] \\
& \hat{Z}_{i j}=\left[\begin{array}{ll}
Z_{i j 1} & Z_{i j 2}
\end{array}\right] \quad \tilde{Z}_{i j}=\left[\begin{array}{ll}
Z_{i j 1} & Z_{i j 2}
\end{array}\right] \\
& \hat{S}_{i j}=\operatorname{diag}\left[\begin{array}{ll}
S_{i j 1} & S_{i j 2}
\end{array}\right] \quad \tilde{S}_{i j} \quad=\operatorname{diag}\left[\begin{array}{ll}
S_{i j 1} & S_{i j 2}
\end{array}\right] \\
& \hat{U}_{i j}=\operatorname{diag}\left[\begin{array}{ll}
U_{i j 1} & U_{i j 2}
\end{array}\right] \quad \tilde{U}_{i j}=\operatorname{diag}\left[\begin{array}{ll}
U_{i j 1} & U_{i j 2}
\end{array}\right] \\
& \hat{W}_{i j}=\operatorname{diag}\left[\begin{array}{ll}
W_{i j 1} & W_{i j 2}
\end{array}\right] \quad \tilde{W}_{i j}=\operatorname{diag}\left[\begin{array}{ll}
W_{i j 1} & W_{i j 2}
\end{array}\right]
\end{aligned}
$$

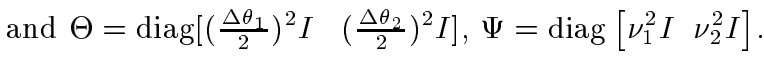

Proposition 2: The set of $\gamma$-suboptimal controllers is nonempty if and only if there exist symmetric $X_{i j 0}, Y_{i j 0}$, $\tilde{X}_{i j}, \hat{Y}_{i j}, \hat{S}_{i j}, \hat{W}_{i j}, \tilde{S}_{i j}, \tilde{W}_{i j}, \hat{U}_{i j}, \tilde{U}_{i j}$ and skew matrices $\hat{T}_{i j}$, $\hat{Z}_{i j}, \tilde{T}_{i j}, \tilde{Z}_{i j}, \hat{V}_{i j}, \tilde{V}_{i j}$ for $\forall i, j$ such that

$$
\begin{gathered}
\hat{S}_{i j}>0, \quad \tilde{S}_{i j}>0, \quad\left[\begin{array}{cc}
\mathcal{X}(\delta) & \gamma^{-1} I \\
\gamma^{-1} I & \mathcal{Y}(\delta)
\end{array}\right]_{i j}>0, \\
{\left[\begin{array}{ccccc}
Q_{1} & Q_{2} & Q_{4} & Y_{i j 0} \hat{C}_{z 1 i j}^{T} & \frac{1}{2} \hat{Y}_{i j}-\hat{Z}_{i j} \\
* & Q_{3} & Q_{5} & -\hat{V}_{i j} & 0 \\
* & * & Q_{6} & \hat{Y}_{i j}^{T} \hat{C}_{z 1 i j}^{T} & 0 \\
* & * & * & -\hat{U}_{i j} & 0 \\
* & * & * & * & -\hat{W}_{i j}
\end{array}\right]<0,} \\
{\left[\begin{array}{ccccc}
Q_{7} & Q_{8} & Q_{10} & X_{i j 0} \tilde{B}_{w 1 i j} & \frac{1}{2} \tilde{X}_{i j}-\tilde{Z}_{i j} \\
* & Q_{9} & Q_{11} & -\tilde{V}_{i j} & 0 \\
* & * & Q_{12} & \tilde{X}_{i j}^{T} \tilde{B}_{w 1 i j} & 0 \\
* & * & * & -\tilde{U}_{i j} & 0 \\
* & * & * & * & -\tilde{W}_{i j}
\end{array}\right]<0,}
\end{gathered}
$$

where each $\delta$ is the vertex of parameter subspace $\mathcal{P}_{i j}$. Here, $Q_{1}=Y_{i j 0} \hat{A}_{i j 0}^{T}+\hat{A}_{i j 0} Y_{i j 0}+\mathcal{I}^{T}\left[\Theta \hat{S}_{i j}+\Psi \hat{W}_{i j}\right] \mathcal{I}+\gamma^{-2} B_{w i j 0} B_{w i j 0}^{T}$ 


$$
\begin{gathered}
-B_{u i j 0} B_{u i j 0}^{T}, \quad Q_{2}=Y_{i j 0} C_{z 1 i j 0}^{T}, \quad Q_{3}=\mathcal{I}^{T} \Theta \hat{U}_{i j} \mathcal{I}-I \\
Q_{4}=Y_{i j 0} \hat{\mathcal{A}}_{i j}^{T}+\hat{A}_{i j 0} \hat{Y}_{i j}-B_{u i j 0} \hat{B}_{u i j}^{T}-\hat{T}_{i j}+\gamma^{-2} B_{w i j 0} \hat{B}_{w i j}^{T}, \\
Q_{5}=C_{z 1 i j 0} \hat{Y}_{i j}, \quad Q_{6}=\hat{Y}_{i j}^{T} \hat{\mathcal{A}}_{i j}^{T}+\hat{\mathcal{A}}_{i j} \hat{Y}_{i j}-\hat{B}_{u i j} \hat{B}_{u i j}^{T}-\hat{S}_{i j} \\
+\gamma^{-2} \hat{B}_{w i j} \hat{B}_{w i j}^{T}, \quad Q_{7}=\tilde{A}_{i j 0}^{T} X_{i j 0}+X_{i j 0} \tilde{A}_{i j 0}+\mathcal{I}^{T}\left[\Theta \tilde{S}_{i j}+\Psi \tilde{W}_{i j}\right] \mathcal{I} \\
+\gamma^{-2} C_{z i j 0}^{T} C_{z i j 0}-C_{y i j 0}^{T} C_{y i j 0}, \quad Q_{8}=X_{i j 0} B_{w 1 i j 0} \\
Q_{10}=\tilde{A}_{i j 0}^{T} \tilde{X}_{i j}+X_{i j 0} \tilde{\mathcal{A}}_{i j}-C_{y i j 0}^{T} \tilde{C}_{y i j}-\tilde{T}_{i j}+\gamma^{-2} C_{z i j 0}^{T} \tilde{C}_{z i j}, \\
Q_{9}=\mathcal{I}^{T} \Theta \hat{U}_{i j} \mathcal{I}-I, \quad Q_{11}=B_{w 1 i j 0}^{T} \tilde{X}_{i j} \\
Q_{12}=\tilde{\mathcal{A}}_{i j}^{T} \tilde{X}_{i j}+\tilde{X}_{i j}^{T} \tilde{\mathcal{A}}_{i j}-\tilde{C}_{y i j}^{T} \tilde{C}_{y i j}-\tilde{S}_{i j}+\gamma^{-2} \tilde{C}_{z i j}^{T} \tilde{C}_{z i j} .
\end{gathered}
$$

Proof: Due to length constraints, we provide a sketch of the derivation. Consider the derivation of Eq. 15. Using the Schur complement, Eq. 12 can be reduced to a $2 \times 2$ block matrix and then to a quadratic inequality (QIE), when premultiplying by $\left[x^{T} w^{T}\right]$ and post-multiplying by $\left[x^{T} w^{T}\right]^{T}$. As in [11], we introduce fictitious variables $p_{k}=\hat{\theta}_{i j k} x$, $q_{k}=\dot{\hat{\theta}}_{i j k} x$ and $r_{k}=\hat{\theta}_{i j k} w$, and formulate this QIE in terms of these new variables. The equality relations between $p_{k}$, $q_{k}, r_{k}$ and $x$ are also transformed to the inequalities using the quadratic relation [11]. Using the $\mathcal{S}$-procedure, QIE and these additional inequalities are then combined to form an augmented quadratic inequality. The derivation of Eq. 14 is similar, but starts with Eq. 11. Furthermore, since $\mathcal{X}_{i j}$ and $\mathcal{Y}_{i j}$ are affine in $\hat{\theta}_{i j}$, Eq. 13 can replace Eq. 10.

\section{Numerical Examples}

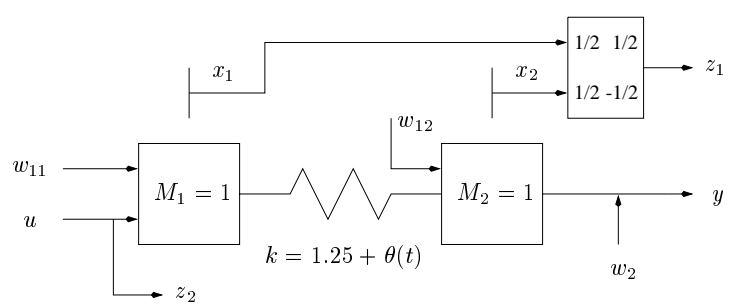

Fig. 2: Block diagram of the system

We will perform a comparative study to investigate several aspects of our approach. The induced $L_{2}$-norm, $\gamma$, is used as a measure of conservatism in various analysis methods. Consider the benchmark problem (see Figure 2) with a timevarying parameter $\theta(t)$ of $|\theta(t)| \leq 0.7$ and $|\dot{\theta}(t)| \leq N t_{r}$, where $t_{r}=1.61 \mathrm{sec}$ is the rise time of the nominal system $\left(t_{r} \approx\right.$ $\left.1.8 / w_{n}\right)$ and $N$ is the normalized bound. It is assumed that $\theta(t)$ and $\dot{\theta}(t)$ are measurable in real time. Then, we can

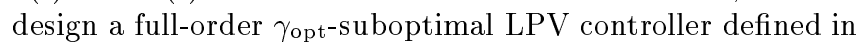
Eq. 3. Note that $\gamma_{\text {opt }}$ means the smallest $\gamma$ achieved by LPV controllers.

We choose 50 points for the normalized bound $N$ between $[0.01,60]$, and for each $N$, find a $\gamma_{\text {opt-suboptimal LPV con- }}$ troller. Because this LPV system is affine $\theta$-dependent, we consider the gridding technique [16] with QALs, named QALs, and our new approach using QPALs with different numbers of partitions, $L \in\{1,2,3,5\}$. As shown in [1], the gridding technique using QALs can lead to less conservative results than other published "convexifying" approaches developed by the same dissipative systems theory. Note, however, that this gridding technique does not guarantee the synthesis result, and thus any post-analysis is required. For each

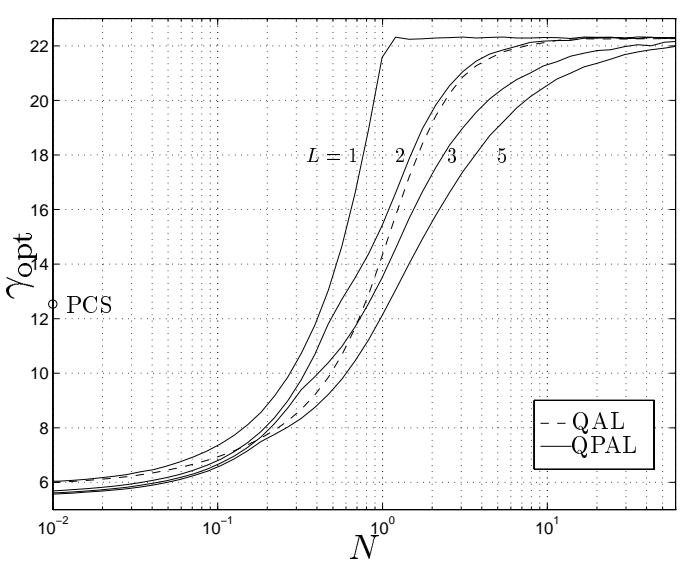

Fig. 3: $\gamma_{\mathrm{opt}}$ 's vs. $N$ of various techniques

technique, we consider two cases: The QAL (or QPAL) indicates a typical case that $X(\theta)$ and $Y(\theta)$ are (piecewise) affine parameter-dependent; The QAL1 (or QPAL1) indicates a special case that $X(\theta)$ is (piecewise-) affine parameterdependent and $Y(\theta)$ is constant. All LMI-related computations were performed with the MATLAB LMI Control Toolbox [8]. The mincx subroutine was used, and ten gridding points were employed for the gridding method.

Figure 3 plots the resulting curves of $\gamma_{\mathrm{opt}}$ 's vs. $N$ of QAL and QPAL techniques. This figure shows the prediction of the guaranteed $\gamma_{\text {opt }}$ from each approach for a broad range of $N$. Note that each of the curves has the same basic shape which indicates that, as expected, the techniques can only guarantee larger $\gamma_{\mathrm{opt}}$ (or worse $L_{2}$ performance) as the rate of change in the parameter increases. This figure shows that as $N \rightarrow 0$, i.e. an unknown but time-invariant parameter, the results of all the cases are much better than that of the Popov controller synthesis (PCS) [3], which uses a special case of the Luré-Postnikov Lyapunov function. These results are confirmed by the fact that the QAL and QPAL use nonlinear parameter-dependent $P_{\mathrm{cl}}(\theta)$ 's (see Eq. 4), thereby reducing conservatism. The large variation of $\gamma_{\mathrm{opt}}$ over the entire range of $N$ implies that typical LFT- $\mu$ approach that only considers $|\dot{\theta}(t)| \rightarrow \infty$ would be very conservative. This figure also shows that our QPAL approach with a small number of partitions ( $L \geq 3$ ) predicts lower $\gamma_{\mathrm{opt}}$ (or better performance) than the QAL approach over almost the entire range of $N$, and thus is less conservative. As discussed in our previous paper [11], this result is due to the fact that our QPAL can use a richer class of parameter-dependent Lyapunov functions. Figure 3 shows that for $N<0.1$, the "convexifying" technique creates little conservatism (see the curve of $L=1$ ), so that the richer Lyapunov function class directly leads to less conservatism $(L \geq 2)$. Figure 3 also shows similar behavior for $N>1$, although the "convexifying" technique becomes conservative $(L=1)$. Furthermore, it is shown that as $L$ increases, our approach predicts even lower values of $\gamma_{\text {opt }}$ 's for intermediate values of $N$. In this example, these improvements in the synthesis are obtained at the cost of polynomially-increased computational time, i.e. a linearly-increased number of LMIs and variables.

To analyze further the benefit of using a parameter-dependent controller, we compare the $\mathcal{H}_{\infty}$-norm of the closed-loop system of several design techniques in case of $N=0$. We select 50 frozen $\theta$ 's in $\left[\begin{array}{ll}-0.7 & 0.7\end{array}\right]$ and construct a perturbed system for each value of $\theta$ and then close the loop with various controllers. We then calculate the nominal $\mathcal{H}_{\infty}$-norm's of 


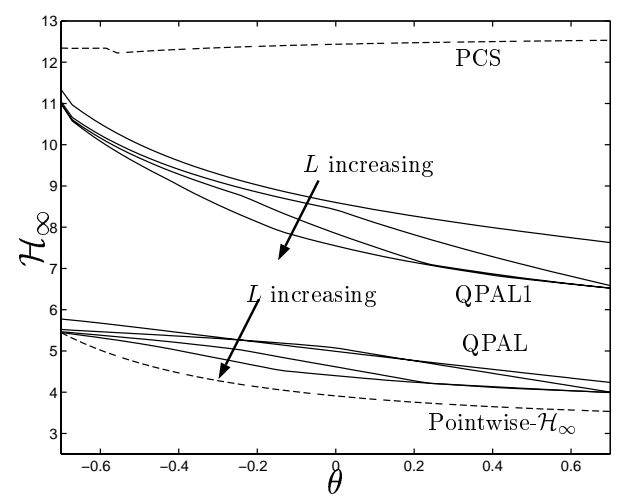

Fig. 4: $\mathcal{H}_{\infty}$-norm's vs. $\theta$ of various techniques

these closed-loop systems. The controllers considered here are the Popov controller [3] and $\gamma_{\mathrm{opt}}$-suboptimal LPV controllers of QPALs. We also include pointwise- $\mathcal{H}_{\infty}$ controllers which are the best LPV controller for this static case. Figure 4 plots $\mathcal{H}_{\infty}$-norm's of the closed-loop systems for four design techniques. This figure shows that the Popov controller yields almost the same performance as the guaranteed $\gamma_{\mathrm{opt}}$ over the entire parameter space. This result is obviously related to the fact that the controller is parameter-independent, thereby being unable to use additional information such as measuring parameters. A similar result is shown in [9]. The performance is the worst because it uses the most restrictive Lyapunov function, as discussed above. This figure also shows that QPAL yields better performance than the worst performance $\left(\gamma_{\mathrm{opt}}\right)$ guaranteed by the synthesis. This is another benefit of using parameter-dependent controllers. As expected, the QPAL1 is obviously more conservative than the QPAL over the entire parameter space. As $L$ increases, the $\mathcal{H}_{\infty}$-norm curve of the QPAL approaches the best achievable performance curve (pointwise- $\mathcal{H}_{\infty}$ ). This result confirms the fact that our QPAL can approximate the required parameterdependent Lyapunov function that produces a less conservative result. However, the gap still remains which is primarily due to the conservatism inherent in the "convexifying" techniques.

\section{Conclusions}

This paper develops the LMI formulation of the induced $L_{2}$ norm synthesis by a nonsmooth dissipation theory using a continuous, quasi-piecewise affine parameter-dependent Lyapunov function. The numerical example shows that this new technique can, with a small number of partitions in the parameter space, provide a less conservative robustness guarantee when compared to previously published methods. While computationally intensive, our approach should help in the investigation of the conservatism of various classes of parameter-dependent Lyapunov functions for different rates of change in the parameter.

\section{Acknowledgments}

The authors would like to thank Dr. Banjerdpongchai for providing the PCS algorithm.

\section{Appendix}

Lemma 2 is more rigorous version than Lemma 1.1 of [11].

Lemma 2: $V(x, \theta)$ is assumed continuous, uniformly Lipschitz in $x$ and $\theta$, respectively. For $\dot{x}$ of Eq. 1 and $\theta \in \mathcal{F}^{2}$,

$$
\begin{aligned}
& D^{+} V(x, \theta) \geq D^{+} V(x, \theta)(L, 0)+D_{+} V(x, \theta)(0, \dot{\theta}), \\
& D^{+} V(x, \theta) \leq D^{+} V(x, \theta)(U, 0)+D^{+} V(x, \theta)(0, \dot{\theta}),
\end{aligned}
$$

where $U(t)=\lim _{h \rightarrow 0}\left[U_{h}=\sup \mathcal{S}_{h}\right], L(t)=\lim _{h \rightarrow 0}\left[L_{h}=\inf \mathcal{S}_{h}\right]$, and $\mathcal{S}_{h}(t)=\{y \mid y=\dot{x}(t+\tau),-h \leq \tau \leq h\}$.

Proof: We provide a sketch of the derivation. In the definition of $D^{+} V(x, \theta)$, add the following terms: $V(x(t+h), \theta(t))-$ $V(x(t+h), \theta(t))$. According to continuity and Lipschitz condition [13],

$$
\begin{aligned}
& D^{+} V(x, \theta) \geq D^{+} V(x, \theta)(v, 0)+D_{+} V(x, \theta)(0, \dot{\theta}), \\
& D^{+} V(x, \theta) \leq D^{+} V(x, \theta)(v, 0)+D^{+} V(x, \theta)(0, \dot{\theta}),
\end{aligned}
$$

where $v=\lim _{h \rightarrow 0} \frac{1}{h} \int_{t}^{t+h} \dot{x}(\tau) d \tau$. The above inequalities are then derived because $L_{h}(t) \leq \frac{1}{h} \int_{t}^{t+h} \dot{x}(\tau) d \tau \leq U_{h}(t)$.

\section{References}

[1] P. Apkarian and R. Adams, "Advanced Gain-Scheduling Techniques for Uncertain Systems," Proc. ACC, 1996

[2] P. Apkarian, P. Gahinet and G. Becker, "Self-Scheduled $\mathcal{H}_{\infty}$ Control of Linear Parameter-Varying Systems: a Design Example," Automatica, 1995, pp1251-1261

[3] D. Banjerdpongchai and J. How, "LMI Synthesis of Parametric Robust $\mathcal{H}_{\infty}$ Controllers," Proc. ACC, 1997

[4] G. Becker and A. Packard, "Robust Performance of Linear Parametrically Varying Systems Using Parametrically-Dependent Linear Feedback," Sys $\&$ Contr. Letters 23, pp205-215, 1994

[5] S. Boyd, L. Ghaoui, E. Feron and V. Balakrishnan, Linear Matrix Inequalities in System and Control Theory, SIAM Studies in Applied Math., 1994

[6] E. Feron, P. Apkarian and P. Gahinet, "S $\mathcal{S}$-Procedure for the Analysis of Control Systems with Parametric Uncertainties via Parameter-Dependent Lyapunov Functions," Proc. $A C C$ 1995, pp968-972

[7] P. Gahinet, P. Apkarian, and M. Chilali, "Affine Parameter-Dependent Lyapunov Functions and Real Parametric Uncertainty," IEEE Trans. on AC, Vol 41, No. 3, pp437-442, 1996

[8] P. Gahinet, A. Nemirovski, A. Laub and M. Chilali, The LMI Control Toolbox, The MathWorks Inc., 1995

[9] A. Helmersson, Methods for Robust Gain Scheduling, $\mathrm{Ph}$.D. Thesis, Linköpings Universitet, 1995

[10] U. Jönsson, Robustness Analysis of Uncertain and Nonlinear Systems, Ph.D. Thesis, Lund Inst. of Tech., 1996

[11] S. Lim and J. How, "Analysis of LPV Systems using a Piecewise Affine Parameter-Dependent Lyapunov Function," Proc. IEEE CDC San Diego, pp978-983, 1997

[12] A. Packard, "Gain Scheduling via Linear Fractional Transformations," Sys\&Contr. Letters, pp79-92, 1994

[13] N. Rouche, P. Habets and M. Laloy, Stability Theory by Liapunov's Direct Method, Applied Math. Sci. 22, Springer-Verlag, 1977

[14] J. Shamma and M. Athans, "Analysis of Gain Scheduled Control for Nonlinear Plants," IEEE Trans. on AC, Vol 35, No.8, pp898-907, 1990

[15] J. Shamma and J. Cloutier, "Gain-Scheduled Missile Autopilot Design Using Linear Parameter Varying Transformations," J. of Guidance, Control and Dynamics, Vol 16, No.2, pp256-263, 1993

[16] F. Wu, Control of Linear Parameter Varying Systems, Ph.D. Dissertation, Univ. of California, Berkeley, 1995 